\title{
SYSTEMS OF INFINITELY MANY LINEAR DIFFERENTIAL EQUATIONS OF INFINITE ORDER, WITH CONSTANT COEFFICIENTS*
}

BY

I. M. SHEFFER $\dagger$

1. INTRODUCTION

The writer has given existence theorems governing a finite system of linear differential equations of infinite order, with constant coefficients. There, the method of attack was one of operators, and by means of suitable differential operators the system was first reduced to a single equation of infinite order, after which certain solutions of this one equation were shown to be solutions of the original system.

Let us adopt the following notation for a differential operator:

$$
A[y] \equiv a_{0} y(x)+a_{1} y^{\prime}(x)+\cdots+a_{n} y^{(n)}(x)+\cdots,
$$

where the $a_{n}$ 's are constants. The system of equations of which we speak has then the form (homogeneous and non-homogeneous)

$$
\begin{array}{ll}
A_{i 1}\left[y_{1}\right]+A_{i 2}\left[y_{2}\right]+\cdots+A_{\text {in }}\left[y_{n}\right]=0 & (i=1,2, \cdots, n) ; \\
A_{i 1}\left[y_{1}\right]+A_{i 2}\left[y_{2}\right]+\cdots+A_{\text {in }}\left[y_{n}\right]=f_{i}(x) & (i=1,2, \cdots, n) .
\end{array}
$$

There are $n$ equations in the $n$ unknown functions $y_{i}(x)$.

Our assumptions, on the basis of which the existence theorems follow, are these:

(i) The $A_{i j}(t)$ are analytic§, $|t|<R$.

(A) (ii)

$$
\Delta(t)=\left|\begin{array}{c}
A_{11}(t) \cdots A_{1 n}(t) \\
\cdots \cdots \cdots \cdot \\
A_{n 1}(t) \cdots A_{n n}(t)
\end{array}\right| \not \equiv 0 .
$$

(iii) The $f_{i}(x)$ are of exponential value $<R$. (For short: of exp. val. $<R$.) And the solutions sought are those functions $y_{i}(x)$ of exp. val. $<R$. We define exponential value as follows:

* Presented to the Society, September 7, 1928; received by the editors in December, 1928.

$\dagger$ National Research Fellow.

I Sheffer, Systems of linear differential equations of infinite order, with constant coefficients, to appear in the Annals of Mathematics. We shall refer to this as Annals Paper I.

$\S$ That is, $A_{i j}(t)=\sum_{n=0}^{\infty} a_{i j, n} n^{n}$ converges, $|t|<R$. 
$f(x)$ is. of exp. val. $\lambda$ if $f(x)$ is entire and $\lim \sup _{n=\infty}\left|f^{(n)}(0)\right|^{1 / n}=\lambda$.

In the present paper we consider the extension to systems of infinitely many equations, and show how such systems can be thrown into the form of systems of contour integral equations in the complex plane. These integral equations are then inverted: we obtain solutions which are themselves contour integrals.* The method employs the Laplace transformation. $\dagger$

In $\$ 2$ we take up the non-homogeneous equation for an infinite system, and in $\$ 3$ we consider the homogeneous case, where solutions are also obtained as contour integrals.

\section{The NON-HOMOgENEOUS CASE, INFINITE SYSTEM}

We consider a system of infinitely many equations in infinitely many unknown functions: $\ddagger$

(III) $A_{i 1}\left[y_{1}\right]+A_{i 2}\left[y_{2}\right]+\cdots+A_{i n}\left[y_{n}\right]+\cdots=f_{i}(x)(i=1,2, \cdots, \infty)$.

We assume that the following conditions are fulfilled:

(i) $A_{i j}(u)$ is analytic, $|u|<R$;

(B) (ii) $f_{i}(x)$ is of exp. val. $\lambda_{i}<\lambda<R$;

(iii) $\left|F_{i}(u)\right| \leqq M$ uniformly in $i$ and in $u$, where $u$ ranges over $|u| \leqq 1 / \lambda$; and

$$
\Delta(u)=\left|\begin{array}{cc}
A_{11} \cdots & A_{1 n} \cdots \\
A_{21} \cdots & A_{2 n} \cdots \\
\cdot & \cdot \\
\cdot & \cdot
\end{array}\right|
$$

is of normal form for every $u$ in $|u|<R$; i.e.,

* The finite case is of course a particular case.

† The Laplace transformation has been used in the complex domain by S. Pincherle, Sur la resolution de l'equation fonctionnelle $\sum h_{\nu} \phi\left(x+\alpha_{\nu}\right)=f(x)$, Acta Mathematica, vol. 48 (1926), pp. 279 304; and in the domain of reals by F. Bernstein and G. Doetsch in a series of papers, of which we cite only two: F. Bernstein and G. Doetsch, Probleme aus der Theorie der Warmeleitung, Mathematische Zeitschrift, vol. 22 (1925), pp. 285-292; G. Doetsch, Die Integrodifferentialgleichungen vom Faltungstypus, Mathematische Annalen, vol. 89 (1923), pp. 192-207.

$\ddagger$ We can equally well consider the system

$$
\sum_{i=-\infty}^{\infty} A_{i j}\left[y_{j}\right]=f_{i}(x)
$$$$
(i=-\infty, \cdots, \infty),
$$

putting on the functions $A_{i j}(u), f_{i}(x)$ the same conditions as we shall lay down for system (III). We arrive at a like existence theorem. For the properties of two-way infinite determinants see von Koch, Sur les déterminants infinis et différentielles linéaires, Acta Mathematica, vol. 16, pp. 217-249. 


$$
\coprod_{i=1}^{\infty}\left|A_{i i}(u)\right| \text { converges and } \sum_{i \neq j}^{\infty}{ }_{i, j=1}\left|A_{i j}(u)\right| \text { converges; }{ }^{*}
$$

(ii) the convergence of the above product and series is uniform in every closed region lying in $|u|<R$;

(iii) $\Delta(u) \neq 0$.

To discuss system (III) we require some preliminary lemmas.

Lemma 1. $\dagger$ If $f(x)=\sum_{0}^{\infty} f_{n} x^{n}$ is of exp. val. $\lambda$, and $F(x)=\sum_{0}^{\infty} F_{n} x^{n}$ where $F_{n}=n ! f_{n}$, then $F(x)$ is analytic in $|x|<1 / \lambda$, and

$$
f(x)=\frac{1}{2 \pi i} \int_{C} \frac{F(u)}{u} e^{x / u} d u
$$

where $C$ is a contour surrounding the origin and lying in $|u|<1 / \lambda$. Conversely, if $F(x)$ has the radius of convergence $1 / \lambda$, and $f(x)$ is given by (1), then $f(x)$ is of exp. val. $\lambda$.

Let us agree, in what follows, to denote by a capital-letter function that function which is related to the corresponding small-letter function as $F(x)$ is to $f(x)$ in Lemma 1 .

LEMma $2 . \ddagger$ If $A(t)$ is analytic, $|t|<R$, and $f(x)$ is of $\exp$. val. $\lambda<R$, then $A[f]$ is entire and is of exp. val. $\leqq \lambda$.

LEMMA $3 . \S$ If $A(t)$ is analytic, $|t|<R$, and $\xi$ is in $|\xi|<R$, then

$$
A\left[e^{\xi x}\right]=A(\xi) e^{\xi x} .
$$

LeMMA 4. If $A(t)$ is analytic, $|t|<R$, and $y(x)$ is of exp. val. $\lambda<R$, then

$$
A[y]=\frac{1}{2 \pi i} \int_{C} \frac{Y(u)}{u} A\left(\frac{1}{u}\right) e^{x / u} d u,
$$

where $C$ is a contour surrounding the origin and lying in $1 / R<|u|<1 / \lambda$.

We see that (3) follows from (1) formally if we use (2). It remains to justify taking the operator $A$ under the integral sign. Now

* It follows that $\Delta(u)$ exists for all $u$ in $|u|<R$. See F. Riesz, Les Systèmes d'Equations Lineaires d une Infinite d'Inconnues (Borel monograph), pp. 24-25.

$\dagger$ Sheffer, Linear differential equations of infinite order, with polynomial coefficients of degree one, to appear in the Annals of Mathematics.

† Sheffer, Annals Paper I.

§Sheffer, Annals Paper I. 


$$
A[y]=\sum_{0}^{\infty} a_{n} y^{(n)}(x)=\sum_{0}^{\infty} a_{n} \cdot \frac{1}{2 \pi i} \int_{C}\left\{\frac{Y(u) e^{x / u}}{u^{n+1}}\right\} d u
$$

and since $\sum_{0}^{\infty} a_{n} / u^{n}$ converges uniformly to $A(1 / u)$ on $C$, term-by-term integration is permissible. This gives (3).

LEMMA 5. If $M(u)$ is continuous on the contour $C$ surrounding the origin, then

$$
y(x)=\frac{1}{2 \pi i} \int_{C} M(u) e^{u x} d u
$$

is of exp. val. @maximum value of $|u|$ on $C$.

We have $|M(u)| \leqq l$ (constant) on $C$. Now use the fact that the power series for $e^{u x}$ is uniformly convergent on $C$.

We consider solutions $\left(y_{1}, y_{2}, \cdots\right)$ of (III) which are of exp. val. $\leqq \mu<R$. If we apply Lemmas 1-4 to (III) we obtain

$$
\sum_{n=0}^{\infty} \frac{1}{2 \pi i} \int_{C} \frac{Y_{n}(u)}{u} A_{i n}\left(\frac{1}{u}\right) e^{x / u} d u=f_{i}(x),
$$

and on integrating term-wise (formally, as yet) we arrive at the system of contour integral equations

$$
\begin{array}{r}
\frac{1}{2 \pi i} \int_{C} \frac{e^{x / u}}{u}\left\{Y_{1}(u) A_{i 1}\left(\frac{1}{u}\right)+\cdots+Y_{n}(u) A_{i n}\left(\frac{1}{u}\right)+\cdots\right\} d u=f_{i}(x) \\
(i=1,2, \cdots),
\end{array}
$$

where $C$ is a contour, surrounding the origin and lying in $1 / R<|u|<1 / \mu$. $^{*}$

Now equations (III') represent the $f_{i}(x)$ as linear expressions in the $Y_{n}(x)$ 's (under an integral). We may then hope to solve by interchanging the rôles of $f, Y$ (and consequently using $F, y$ ). We look, then, for a solution of (III) in the form

* If we deal with the case of a finite system: (b) $\sum_{i=1}^{n} A_{i j}\left[y_{j}\right]=f_{i}(x), i=1, \cdots, n$, the step from (a) to $\left(\mathrm{III}^{\prime}\right)$ is no longer merely formal, and we have the following theorem of equivalence: of

THEOREM. If $\left(y_{1}, \cdots, y_{n}\right)$ of exp. val. $\leqq \mu<R$ is a solution of $(\mathrm{b})$, then $\left(Y_{1}, \cdots, Y_{n}\right)$ is a solution

$$
\frac{1}{2 \pi i} \int_{C}\left\{\sum_{j=1}^{n} Y_{j}(u) A_{i j}(1 / u)\right\} \frac{e^{x / \mu}}{u} d u=f_{i}(x) \quad(i=1, \cdots, n)
$$

if the contour $C$, surrounding the origin, is chosen to lie in $1 / R<|t|<1 / \mu$. Conversely, if $\left(Y_{1}, \cdots, Y_{n}\right)$, analytic in $|t|<1 / \mu$ (where $\mu<R)$, is a solution of $\left(\mathrm{b}^{\prime}\right)$, then $\left(y_{1}, \cdots, y_{n}\right)$ is a solution of (b) and is of $\exp$. val. $\leqq \mu$. 


$$
\begin{aligned}
y_{i}(x)=\frac{1}{2 \pi i} \int_{\Gamma} \frac{e^{x / u}}{u}\left\{F_{1}(u) \Lambda_{i 1}\left(\frac{1}{u}\right)\right. & +\cdots+F_{n}(u) \Lambda_{i n}\left(\frac{1}{u}\right) \\
& +\cdots\} d u \quad(i=1,2, \cdots),
\end{aligned}
$$

the contour $\Gamma$ and the functions $\Lambda_{i j}(u)$ to be determined.

If we substitute the values (4) into (III) formally, we find the equations

$$
\begin{gathered}
f_{i}(x)=\frac{1}{2 \pi i} \int_{\Gamma} \frac{e^{x / u}}{u}\left\{F_{1}(u) \sum_{j=1}^{\infty} A_{i j}\left(\frac{1}{u}\right) \Lambda_{j 1}\left(\frac{1}{u}\right)+\cdots\right. \\
\left.\quad+F_{n}(u) \sum_{j=1}^{\infty} A_{i j}\left(\frac{1}{u}\right) \Lambda_{j n}\left(\frac{1}{u}\right)+\cdots\right\} d u .
\end{gathered}
$$

A sufficient condition (see Lemma 1) that these relations hold, $i=1,2, \cdots$, is that the coefficient of $F_{n}(u)$ be identically unity or zero according as $n=i$ or $n \neq i$. This gives rise to the following infinite systems of linear equations in the unknowns $\Lambda_{j n}(u)$ :

$$
\sum_{j=1}^{\infty} A_{i j}(u) \Lambda_{j n}(u)=\delta_{i n}=\left\{\begin{array}{l}
0, i \neq n, \\
1, i=n .
\end{array}\right.
$$

Here $i=1,2, \cdots$, while $n$ may be given a fixed value. As we vary $n$ we obtain different systems of equations, but they all have the same determinant:

$$
\Delta(u)=\left|\begin{array}{cc}
A_{11} \cdots & A_{1 n} \cdots \\
A_{21} \cdots & A_{2 n} \cdots \\
\vdots & \vdots
\end{array}\right|
$$
that

On solving systems (5) in the customary way (by determinants) we find

$$
\Lambda_{j n}(u)=\frac{\Delta_{j n}(u)}{\Delta(u)},
$$

where

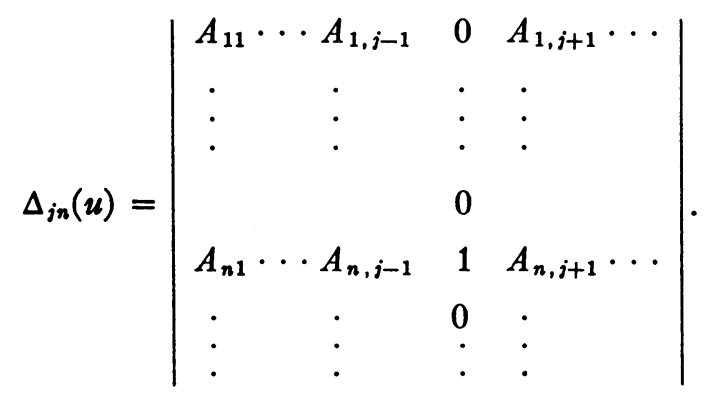


Returning to (4) we see that the brace in the integrand can be written as a determinant, so that we have

$$
y_{i}(x)=\frac{1}{2 \pi i} \int_{\Gamma} \frac{e^{x / u}}{u \Delta(1 / u)} \Delta_{i}(u ; F) d u \quad(i=1,2, \cdots, \infty),
$$

where

$$
\Delta_{i}(u ; F)=\left|\begin{array}{c}
A_{11}\left(\frac{1}{u}\right) \ldots A_{1, i-1}\left(\frac{1}{u}\right) F_{1}(u) A_{1, i+1}\left(\frac{1}{u}\right) \ldots \\
A_{21}\left(\frac{1}{u}\right) \ldots A_{2, i-1}\left(\frac{1}{u}\right) F_{2}(u) A_{2, i+1}\left(\frac{1}{u}\right) \ldots \\
\ldots \ldots \ldots \ldots \ldots \ldots \ldots \ldots \ldots \ldots
\end{array}\right| .
$$

We choose the contour $\Gamma$ to surround the origin and to lie in $1 / R<|u|<1 / \lambda$.

The above work has been formal. It can be made rigorous on the basis of our groups of assumptions (B) and (C). But we need only the final result (9). In fact, we have

THEOREM. The functions $y_{i}(x)$ given by (9) satisfy system (III) and are of $\exp$. val. $\leqq R^{\prime}<R$.

From conditions (B) and (C) it follows that $\Delta(1 / u), \Delta_{i}(u ; F)$ converge uniformly* and absolutely on $\Gamma$. The functions $y_{i}(x)$ as given by (9) are then of exp. val. $\leqq R^{\prime}=1 / r$ where $r$ is the minimum distance of the origin from $\Gamma$ (Lemma 5). Then, $R^{\prime}<R$. Now

$$
A_{i j}\left[y_{i}\right]=\frac{1}{2 \pi i} \int_{\Gamma} \Delta_{j}(u ; F) A_{i j}(1 / u) \frac{e^{x / u}}{u \Delta(1 / u)} d u
$$

so that

$$
\sum_{j=1}^{\infty} A_{i j}\left[y_{j}\right]=\frac{1}{2 \pi i} \int_{\Gamma} \frac{e^{x / u}}{u \Delta(1 / u)}\left\{\sum_{j=1}^{\infty} A_{i j}(1 / u) \Delta_{j}(u ; F)\right\} d u,
$$

provided the series in the brace is uniformly convergent on $\Gamma$. Let us establish this result. We can write

$$
\Delta_{i}(u ; F)=\sum_{j=1}^{\infty} \Delta_{i j}(1 / u) F_{j}(u),
$$

where $\Delta_{i j}$ is given by (8), and this series is absolutely and uniformly convergent on $\Gamma$; furthermore, the sum of the series of absolute values is bounded

* With respect to uniformity, here and later, see von Koch, loc. cit., pp. 238-244, and Riesz, loc. cit., pp. 39-40. The absolute convergence is a well known result: Riesz, loc. cit., p. 26. 
uniformly* in $i: \sum_{j=1}^{\infty}\left|\Delta_{i j}(1 / u) F_{j}(u)\right| \leqq K$, independent of $i$. From this follows the convergence, and uniform convergence on $\Gamma$, of the series $\sum_{j, k}\left|\Delta_{j k}(1 / u) A_{i j}(1 / u) F_{k}(u)\right|$. For

$$
\sum_{j, k}\left|\Delta_{j k} A_{i j} F_{k}\right|=\sum_{j}\left|A_{i j}\right|\left\{\sum_{k}\left|F_{k} \Delta_{j k}\right|\right\} \leqq K \sum_{j}\left|A_{i j}\right|,
$$

and this last series converges. We may then sum the double series $\sum_{j, k} \Delta_{j k}(1 / u) A_{i j}(1 / u) F_{k}(u)$ in any manner, and one such summation yields

$$
\sum_{j} A_{i j}\left\{\sum_{k} F_{k} \Delta_{j k}\right\}=\sum_{j} A_{i j}(1 / u) \Delta_{j}(u ; F) .
$$

The uniformity of convergence is not destroyed, so that $(\alpha)$ is established.

But another mode of summing gives $\sum_{k} F_{k}\left\{\sum_{j} A_{i j} \Delta_{j k}\right\}$, and for the brace we have the well known relation $\dagger$

Hence

$$
\sum_{j=1}^{\infty} A_{i j}(1 / u) \Delta_{j k}(1 / u)= \begin{cases}0, & i \neq k \\ \Delta(1 / u), & i=k\end{cases}
$$

$$
\sum_{j} A_{i j}(1 / u) \Delta_{j}(u ; F)=F_{i}(u) \Delta(1 / u),
$$

so that we can rewrite $(\alpha)$ to give

$$
\sum_{j=1}^{\infty} A_{i j}\left[y_{j}\right]=\frac{1}{2 \pi i} \int_{\Gamma} F_{i}(u) \frac{e^{x / u}}{u} d u .
$$

By Lemma 1 the right hand member is precisely $f_{i}(x)$. That is, $\left(y_{1}, \cdots\right.$, $\left.y_{n}, \ldots\right)$ is a solution of (III).

Let us return to the system of contour integral equations (III'). The set $\left(Y_{1}, \cdots, Y_{n}, \cdots\right)$ which corresponds to $\left(y_{1}, \cdots, y_{n}, \cdots\right)$ as defined by (9) is given by

$$
Y_{i}(x)=\frac{1}{2 \pi i} \int_{\mathrm{r}} \frac{\Delta_{i}(u ; F) d u}{(u-x) \Delta(1 / u)} \quad(i=1,2, \cdots) .
$$

Corollary. The functions $Y_{i}(x)$ defined by (12) satisfy system (III').

Equations (a) follow from system (III), since the $y_{i}(x)$ are of exp. val. $\leqq \mu<R$, where $\mu$ is properly chosen. If now we substitute (12) into (a) we obtain

\footnotetext{
* Riesz, loc. cit., p. 26.

† Riesz, loc. cit., p. 27.

$\ddagger$ For $y_{i}$ and $Y_{i}$ are related in the same way that $e^{x / u}$ and $1 /(1-x / u)$ are related.
} 


$$
\sum_{n=0}^{\infty}\left(\frac{1}{2 \pi i}\right)^{2} \int_{C} \int_{\Gamma} \frac{e^{x / u} A_{i n}(1 / u)}{u(t-u) \Delta(1 / t)} \Delta_{n}(t ; F) d u d t=f_{i}(x) .
$$

But $\sum_{n=0}^{\infty} A_{\text {in }}(1 / u) \Delta_{n}(t ; F)$ converges uniformly on $C, \Gamma$, so that we can integrate term-wise. This gives (III').

Remark. The assumptions (B) and (C) have been used only to establish the validity of the formal processes. These hypotheses may be replaced by others. In fact we have the*

THEOREM. The functions $y_{i}(x)$ of (9) satisfy system (III) and are of exp. val. $\leqq R^{\prime}<R$, when assumptions (B), (C) are replaced by

(i) $A_{i j}(t)$ analytic, $|t|<R$;

(B') (ii) $f_{i}(x)$ is of exp. val. $\lambda_{i}<\lambda<R$;

(iii) $\sum_{i=1}^{\infty}\left|F_{i}(u)\right|^{2}$ converges uniformly in $|u| \leqq 1 / \lambda$; and

(i) in the determinant $\Delta(u)$, the series

$$
\sum_{i \neq j}^{\infty}\left|A_{i j}(u)\right|^{2}
$$

and the product $\prod_{i=1}^{\infty}\left|A_{i i}(u)\right|$ converge for every $u$ in $|u|<R$ (it follows that $\Delta(u)$ is absolutely converent $\dagger)$;

(ii) the convergence of the above product and series is uniform in every closed region lying in $|u|<R$;

$$
\Delta(u) \not \equiv 0 .
$$

It is only necessary to go over the proof of the preceding theorem, observing that each step can be justified under the new hypotheses.

\section{The homogeneous case}

We consider now the homogeneous system

$$
A_{i 1}\left[y_{1}\right]+A_{i 2}\left[y_{2}\right]+\cdots+A_{i n}\left[y_{n}\right]+\cdots=0 \quad(i=1,2, \cdots),
$$

with assumptions $(\mathrm{B}),(\mathrm{C})$ or assumptions $\left(\mathrm{B}^{\prime}\right),\left(\mathrm{C}^{\prime}\right)$, and look for a solution similar to the expressions (9). We set

* I owe to Professors Hille and Tamarkin the observation that the methods already employed will be valid under the conditions $\left(\mathrm{B}^{\prime}\right),\left(\mathrm{C}^{\prime}\right)$.

† See H. von Koch, Sur le convergence des déterminants infinis, Rendiconti del Circolo Matematico di Palermo, vol. 28 (1909), pp. 255-266.

$\ddagger$ For the theory of determinants which are absolutely convergent, see the paper of von Koch which has just been cited. 
(13)

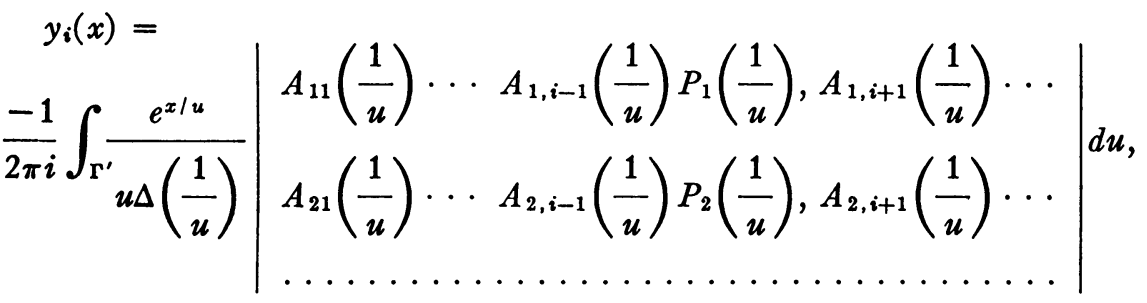

where $\Gamma^{\prime}$ is a small contour surrounding $t=1 / \xi$, with $\Delta(\xi)=0$ and $|\xi|<R$, and $P_{1}(u), P_{2}(u), \cdots$ are arbitrary polynomials* which vanish at $u=0$.

On making the transformation $t=1 / u$ we obtain

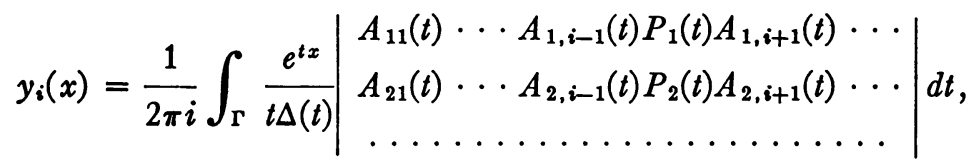

where $\Gamma$ is a small contour surrounding $t=\xi$ and containing no other zero of $\Delta(t)$. We find that $\dagger$

$$
A_{i 1}\left[y_{1}\right]+\cdots+A_{i n}\left[y_{n}\right]+\cdots=\frac{1}{2 \pi i} \int_{\Gamma} P_{i}(t) \frac{e^{t x}}{t} d t
$$

and this is zero since the integrand is analytic in and on $\Gamma$. Consequently, $\left(y_{1}, y_{2}, \cdots\right)$ as given by (14) is a solution of system (IV); and to each zero $t=\xi$ of $\Delta(t)$ (in $|t|<R$ ) there corresponds one or more solutions of (IV). $\ddagger$

* They are also subject to the condition that they make the determinant in which they appear uniformly convergent on $\Gamma^{\prime}$. This will be the case if $\left|P_{i}(u)\right| \leqq N$ uniformly in $i$ and $u$, where $u$ ranges over any closed region in $|u|<R$ (case (B)) or if $\sum_{i=1}^{\infty}\left|P_{i}(u)\right|^{2}$ converges uniformly in every closed region in $|u|<R$ (case $\left.\left(\mathrm{B}^{\prime}\right)\right)$.

$\dagger$ Convergence questions are treated as in $\$ 2$.

$\ddagger$ In Sheffer, Annals Paper I, we show that for a finite system there are precisely $s(\xi)$ linearly independent solutions corresponding to the zero $t=\xi$ of $\Delta(t)$ (in $|t|<R$ ), where $s(\xi)$ is the order of the zero.

PrINCETON UNIVERSITY, Princeton, N. J. 